\title{
Salmonella Meningitis Presenting With Multiple Microabscesses in the Brain in a Young Infant: A Case Report
}

\author{
Basheer Ahmed ${ }^{\mathrm{a}}$, Abdul Elah Al Jarallah ${ }^{\mathrm{b}, \mathrm{c}}$, Abdulrahman Asiri ${ }^{\mathrm{b}}$, Ali Al Hanash ${ }^{\mathrm{b}}$, \\ Ali Al Maely ${ }^{\mathrm{b}}$, Hassan Ogran ${ }^{\mathrm{b}}$, Abdullah Muzallaf ${ }^{\mathrm{a}}$, Ahmed Al Jarie ${ }^{\mathrm{a}}$
}

\begin{abstract}
We report the case of a 4-month-old girl who presented with salmonella meningitis with multiple microabscesses in the brain, and was managed with appropriate intravenous antibiotics for 4 weeks with clearance of microabscesses on repeated imaging.
\end{abstract}

Keywords: Infant; Brain microabscesses; Meningitis; Salmonella

\section{Introduction}

Salmonella is a common cause of community-acquired gastrointestinal infection; however, they rarely do cause severe infection in the form of bacteremia and meningitis. Salmonella meningitis predominantly affects the neonates and infants, and it is associated with a lot of complications with high morbidity and mortality $[1,2]$. Salmonella meningitis usually presents as a focal intracranial abscess or intraventricular hemorrhage [3], but presenting as multiple microabscesses in the brain is not frequently seen.

\section{Case Report}

Our patient was a 4-month-old Saudi girl, a product of fullterm, normal vaginal delivery. She was apparently normal up to 4 months of age and had received only the primary vaccination given at birth and no subsequent vaccinations were given. She presented with fever for 3 days, continuous, relived with anti-pyretic and abnormal movements once on the day of presentation, involving all four limbs, tonic clonic, lasting for about a minute with no history of loss of consciousness. She was on breast feeds.

Manuscript accepted for publication February 04, 2016

a Abha Maternity and Pediatric Hospital, Abha, Saudi Arabia ${ }^{b}$ College of Medicine, King Khalid University, Abha, Saudi Arabia ${ }^{\mathrm{c}}$ Corresponding Author: Abdul Elah Al Jarallah, College of Medicine, King Khalid University, Abha, Saudi Arabia. Email: AAJarallah9@gmail.com

doi: http://dx.doi.org/10.14740/ijcp236w
On presentation to our emergency room, she was looking sick, drowsy with bulging anterior fontanel, heart rate of 140 beats/min, blood pressure of 104/60 $\mathrm{mm} \mathrm{Hg}$, respiratory rate of 60 breaths $/ \mathrm{min}, \mathrm{O}_{2}$ saturation of $94 \%$ in room air, length of 54 $\mathrm{cm}$, weight of $4.7 \mathrm{~kg}$ and head circumference of $39 \mathrm{~cm}$. Capillary filling time was $2 \mathrm{~s}$. On systemic examination, central nervous system showed the pupils were unequal in size, the right pupil was dilated, the left pupil was normal in size and both were reacting to light. Motor examination of upper and lower limbs showed increased tone and reflexes on the right side compared to the left side, with bilateral up going plantar. Modified Glasgow coma score was 8.

Other systemic examinations, namely, chest, abdomen and pelvis were unremarkable. She was admitted to pediatric ICU in view of altered level of consciousness in an infant with probable meningitis, where she was intubated and ventilated. Urgent $\mathrm{CT}$ brain was done which was reported as normal. Initial lab works showed hemoglobin of $8.7 \mathrm{~g} / \mathrm{dL}$, WBC of 6.66 $\times 10^{3}$, and platelets of $297 \times 10^{3}$. Serum electrolytes, urea, creatinine, liver function test, serum calcium, magnesium and phosphate were within normal limits.

Lumbar puncture was done, CSF was clear, and CSF cell count showed WBC of 1,500 cell $/ \mathrm{mL}$ with $70 \%$ neutrophils and $30 \%$ lymphocytes, and RBC of 0 - 1 cells/HPF. CSF glucose was 0 and CSF protein was $170 \mathrm{mg} / \mathrm{dL}$. Patient was empirically started on cefotaxime and gentamicin, and later CSF culture and blood culture were reported to be positive for salmonella, sensitive to ampicillin, ceftriaxone, cefepime, ceftazidime and meropenem. So ampicillin was added to the above antibiotics which the patient was receiving.

Patient continued to be highly febrile and did not show any neurological improvement and her repeat blood cultures came negative, so repeat CT brain was done which showed multiple microabscesses in the brain with no evidence of hydrocephalus. Repeat lumbar puncture was done which was still positive for salmonella, so patient was commenced on meropenem and her ampicillin and cefotaxime was stopped. She started to respond as the fever subsided within $48 \mathrm{~h}$ of starting meropenem and repeat lumbar puncture after a week of meropenem showed the CSF culture was negative.

Neurosurgical consultation was sought in regards to multiple microabscesses but as per the neurosurgeon, no intervention can be done and the patient needed to be managed conservatively with intravenous antibiotics. Patient showed clinical im- 
provement, so she was weaned off the ventilator and was shifted to the ward. Her modified Glasgow coma score improved to 13 and she was continued on meropenem. Nasogastric feeds were started and she tolerated it well. Three weeks later, US cranium was done which did not pick up the microabscesses and there was mild hydrocephalus, so CT brain was repeated which showed there was complete resolution of microabscesses and there was newly developed dilatation of the ventricular system, mainly involving the supratentorial ventricular system with transependymal CSF leak denoting significant increases in intraventricular pressure.

Subsequently, neurosurgical consultation was sought. Patient had a ventriculo-peritoneal shunt inserted, and her modified Glasgow coma score increased to 15 , and she received meropenem for a total duration of 4 weeks. She was also commenced on active physiotherapy for her right-sided weakness.

At the time of discharge, patient tolerated oral feeds well, cranial nerves were intact, and she had mild spasticity on the right upper and lower limbs, for which she was on active physiotherapy.

\section{Discussion}

Salmonella meningitis has been reported following contact with reptiles, following ingestion of infected breast milk of mothers infected with salmonella, and having contact with stools of salmonella carrier mothers $[4,5]$. There are a lot of salmonella serovars reported like salmonella enterica, paratyphi, infantis, houtenae, Agona and typhimurium [6, 7].

Salmonella meningitis leads to a lot of neurological complications like hydrocephalus, subdural empyema, ventriculitis, cerebral abscesses, convulsive disorder, coma and spastic paralytic alterations. It is associated with increased mortality $[8,9]$.

Third generation cephalosporins and quinolones are the initial treatment in many of the reported cases; chloramphenicol, cotrimoxazole and ampicillin were also used whether alone or added to one of the previously mentioned antibiotics. For patients with cerebral abscesses, neurosurgical drainage is indicted $[3,10]$.

Duration of antimicrobial therapy is an important factor in prevention of recurrence as there are a few reported incidence of recurrence of salmonella meningitis in patients whose antibiotics were stopped after 2 - 3 weeks.

When patients are presenting with cerebral abscess which cannot be drained, we recommend treating with broad spectrum antibiotics and giving antibiotics for at least 4 - 5 weeks duration or until all the abscesses in the brain have disappeared [2].

\section{References}

1. Punpanich W, Netsawang S, Thippated C. Invasive salmonellosis in urban Thai children: a ten-year review. Pediatr Infect Dis J. 2012;31(8):e105-110.

2. Emejuru O, Jayam-Trouth A. Salmonella meningitis in infancy. J Natl Med Assoc. 1988;80(7):824-825, 829.

3. Mahapatra AK, Pawar SJ, Sharma RR. Intracranial Salmonella infections: meningitis, subdural collections and brain abscess. A series of six surgically managed cases with follow-up results. Pediatr Neurosurg. 2002;36(1):813.

4. Ricard C, Mellentin J, Ben Abdallah Chabchoub R, Kingbede P, Heuclin T, Ramdame A, Bouquet A, et al. [Salmonella meningitis in an infant due to a pet turtle]. Arch Pediatr. 2015;22(6):605-607.

5. Geiseler PJ, Nelson KE, Reddi KT. Unusual aspects of Salmonella meningitis. Clin Pediatr (Phila). 1980;19(10):699-703.

6. Mahalakshmi R, Rajeshbabu B, Mohan R, Balakumaran D, Venkataraman P, Ponnurangam Nagarajan V. Salmonella paratyphi B meningitis in an infant. Australas Med J. 2013;6(7):350-353.

7. Ceyhan M, Ertugrul M, Gogus S, Secmeer G. [Salmonella meningitis]. Mikrobiyol Bul. 1983;17(3):186-190.

8. Totan M, Kucukoduk S, Dagdemir A, Dilber C. Meningitis due to Salmonella in preterm neonates. Turk J Pediatr. 2002;44(1):45-48.

9. Workman MR, Price EH, Bullock P. Salmonella meningitis and multiple cerebral abscesses in an infant. Int $\mathbf{J}$ Antimicrob Agents. 1999;13(2):131-132.

10. Low LC, Lam BC, Wong WT, Chan-Lui WY, Yeung CY. Salmonella meningitis in infancy. Aust Paediatr J. $1984 ; 20(3): 225-228$. 